\title{
Critical analysis of vacancy-induced magnetism in monolayer and bilayer graphene
}

\author{
J. J. Palacios and F. Ynduráin \\ Departamento de Física de la Materia Condensada, Universidad Autónoma de Madrid, Cantoblanco, Madrid 28049, Spain
}

(Received 29 March 2012; revised manuscript received 22 May 2012; published 25 June 2012)

\begin{abstract}
The observation of intrinsic magnetic order in graphene and graphene-based materials relies on the formation of magnetic moments and a sufficiently strong mutual interaction. Vacancies are arguably considered the primary source of magnetic moments. Here we present an in-depth density functional theory study of the spin-resolved electronic structure of (monoatomic) vacancies in graphene and bilayer graphene. We use two different methodologies: supercell calculations with the SIESTA code and cluster-embedded calculations with the ALACANT package. Our results are conclusive: The vacancy-induced extended $\pi$ magnetic moments, which present long-range interactions and are capable of magnetic ordering, vanish at any experimentally relevant vacancy concentration. This holds for $\sigma$-bond passivated and unpassivated reconstructed vacancies, although, for the unpassivated ones, the disappearance of the $\pi$ magnetic moments is accompanied by a very large magnetic susceptibility. Only for the unlikely case of a full $\sigma$-bond passivation, preventing the reconstruction of the vacancy, a full value of $1 \mu_{B}$ for the $\pi$ extended magnetic moment is recovered for both monolayer and bilayer cases. Our results put on hold claims of vacancy-induced ferromagnetic or antiferromagnetic order in graphene-based systems, while still leaving the door open to $\sigma$-type paramagnetism.
\end{abstract}

DOI: 10.1103/PhysRevB.85.245443

PACS number(s): 73.22.Pr

\section{INTRODUCTION}

According to theory, the existence of intrinsic (without invoking foreign species) magnetism in graphene and graphenebased materials should be the rule rather than the exception. Besides trivial paramagnetism associated with $\sigma$ dangling bonds of undercoordinated $\mathrm{C}$ atoms, a more interesting $\pi$ magnetism should arise at zigzag edges: ${ }^{1-10}$ bulk defects such as vacancies, ${ }^{11-19}$ or grain boundaries, ${ }^{20}$ which either appear naturally or can be created in a more or less controlled manner. However, undisputed experimental evidence of magnetic order remains elusive since early claims of observation of ferromagnetism in irradiated graphite ${ }^{21,22}$ and graphene. ${ }^{23}$ Recent claims based on transport in untreated graphene ${ }^{24}$ and local probe measurements ${ }^{20,25,26}$ seem more solid, but not entirely free from controversy. ${ }^{27}$ The reasons why the observation of ferromagnetism in graphene and graphene derivatives is so elusive, even at low temperatures, are still unclear but can generically be traced back to two facts: 1) the magnetic instability leading to the appearance of magnetic moments can be superseded by structural (Jahn-Teller) instabilities or unwanted passivation by foreign species, and 2) the underlying antiferromagnetic correlations inherent to graphene favor this type of magnetic order over ferromagnetism even if the magnetic moments truly exist.

Graphene represents the paradigm of bipartite lattices. At the heart of the bipartite nature lies the reason why some graphene derivatives result in half-filled $\pi$ states at the Fermi energy which spin-split due to electron-electron interactions. When these interactions are restricted to be local, as described, e.g., by a one-orbital Hubbard model, and the electron-hole symmetry is exactly preserved, the existence of a magnetic ground state with total spin $S=\left|N_{A}-N_{B}\right| / 2$ is guaranteed by a theorem by Lieb, ${ }^{28}$ where $N_{A}-N_{B}$ is the difference between the number of atoms in each sublattice, i.e., the sublattice imbalance. This imbalance appears whenever graphene is cut or grown into triangular shapes bounded by zigzag edges ${ }^{7}$ or, conversely, when $\mathrm{C}$ atoms are removed from bulk graphene, creating vacancies ${ }^{13}$ or voids ${ }^{15}$ with similar triangular shapes. Interestingly, even when $N_{A}-N_{B}=0$ as is the case in zigzag nanoribbons, ${ }^{1-6}$ large hexagonal graphene nanoflakes with zigzag edges, ${ }^{7,8}$ or voids of similar shape in bulk graphene, ${ }^{15}$ magnetic solutions may appear but always with an envelope antiferromagnetic order on top of a local ferromagnetic order. Smaller structures with $N_{A}-N_{B}=0$ are not magnetic as, e.g., recent work on divacancies in graphene ${ }^{29}$ shows.

In between Lieb's theorem and the observation of magnetic fingerprints in graphene-based systems stands a number of assumptions easily overlooked, namely: (i) that the bipartite atomic structure is preserved, (ii) that hydrogenation or passivation of the extended $\pi$ states does not occur, (iii) that the Hubbard model is a good approximation to describe graphene $\pi$ interactions, and (iv) that the substrate does not play a significant role. Despite that both chemical synthesis and physical approaches are making progress into the creation of locally or globally imbalanced graphene structures, it still remains a remarkably challenging task. The second condition is a major experimental challenge since it is difficult to avoid full passivation of the edges ${ }^{30}$ (not to mention to achieve the often implicit selective passivation of the $\sigma$ bonds, while avoiding that of the $\pi$ states), at least under conditions compatible with standard magnetic measurements. Moreover, if passivation is completely avoided in ultrahigh vacuum conditions, the equilibrium atomic structure may develop a reconstruction that ruins the first condition and that, energy wise, competes favorably with the magnetic instability. ${ }^{31,32}$ Substrates may prevent this reconstruction from taking place, ${ }^{33}$ but it is unclear whether or not they always respect the magnetic instability. Finally, while the (mean-field) Hubbard model has shown its reliability in reproducing results obtained with more sophisticated approximations [typically density functional theory $\left.(\mathrm{DFT})^{7}\right]$, the comparison has only been carried out in the most favorable situation, namely, saturating the $\sigma$ bonds with $\mathrm{H}$, thus avoiding unwanted lattice reconstructions. The extent 


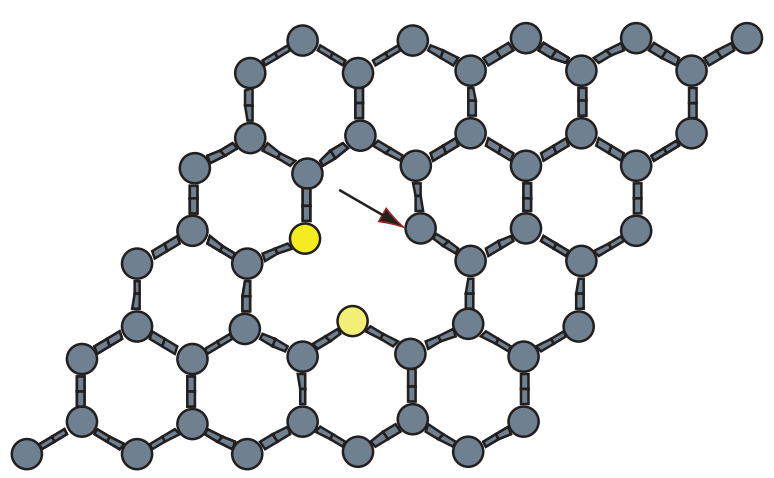

FIG. 1. (Color online) Atomic structure around a single atom vacancy in graphene. The arrow indicates the atom with the dangling $\sigma$ bond, and the colored atoms are those rebonded.

to which the unsaturated $\sigma$ bonds or lattice reconstructions may invalidate the use of the one-orbital Hubbard model remains largely unexplored.

While the magnetic instability at zigzag edges is under present theoretical and experimental scrutiny, the natural hosts of magnetism in bulk, vacancies, have not received due critical attention so far. Single-atom vacancies in bulk graphene are the simplest structures complying, in principle, with the conditions for the appearance of extended $\pi$ magnetic moments, both in monolayer ${ }^{12-16,18,19,34}$ and multilayer graphene. ${ }^{35-37}$ When $\mathrm{H}$ saturation of the dangling bonds (left upon removal of a $\mathrm{C}$ atom) prevents the Jahn-Teller reconstruction of the vacancy, the value of the induced magnetic moment is expected to be $1 \mu_{B}$ for the $\pi$ electrons plus 0 or $1 \mu_{B}$ for the $\sigma$ bonds, depending on whether three or two $\mathrm{H}$ atoms are available for saturation, respectively. Discrepancies, however, can be found in the literature regarding the actual value of the magnetic moment when a single $\mathrm{H}$ (or no $\mathrm{H}$ at all) saturates a dangling bond and the vacancy reconstructs (see Fig. 1). Values for the $\pi$ magnetic moment ranging from $\approx 0.0 \mu_{B}$ to $\approx 1.0 \mu_{B}$ have been reported in this case. ${ }^{11,12,14,16,19,38}$

To illustrate the source of the discrepancy we show in Fig. 2 the electronic structure of a single vacancy in a graphene monolayer in two different instances. In panel (a) we consider the three $\sigma$ dangling bonds, left by the removal of a $\mathrm{C}$ atom, saturated with $\mathrm{H}$ atoms, whereas in panel (b) we consider no $\mathrm{H}$ passivation. In both cases a full atomic relaxation is carried out. A schematic of the atomic structure in the second case is shown in Fig. 1, which is similar to the ones previously reported in the literature. . $^{11,12,14,16,19,38}$ The differences in the electronic structure between both situations are remarkable. In the former case the trigonal atomic symmetry is maintained, and we obtain two well separated spin minority and spin majority $\pi$ bands (dispersive due to the supercell periodicity) near the Fermi level situated at the Dirac point. The magnetic moment associated with the $\pi$ orbitals is actually $1 \mu_{B}$. In Fig. 2(b), aside from the appearance of a $\sigma$ band at $-0.75 \mathrm{eV}$, the situation is different. The $\pi$ bands overlap in energy close to the Fermi level and, therefore, the magnetic moment is smaller than $2 \mu_{B}$ (1.71 in this case). Importantly, the Dirac point lies above the Fermi level at around $0.25 \mathrm{eV}$.
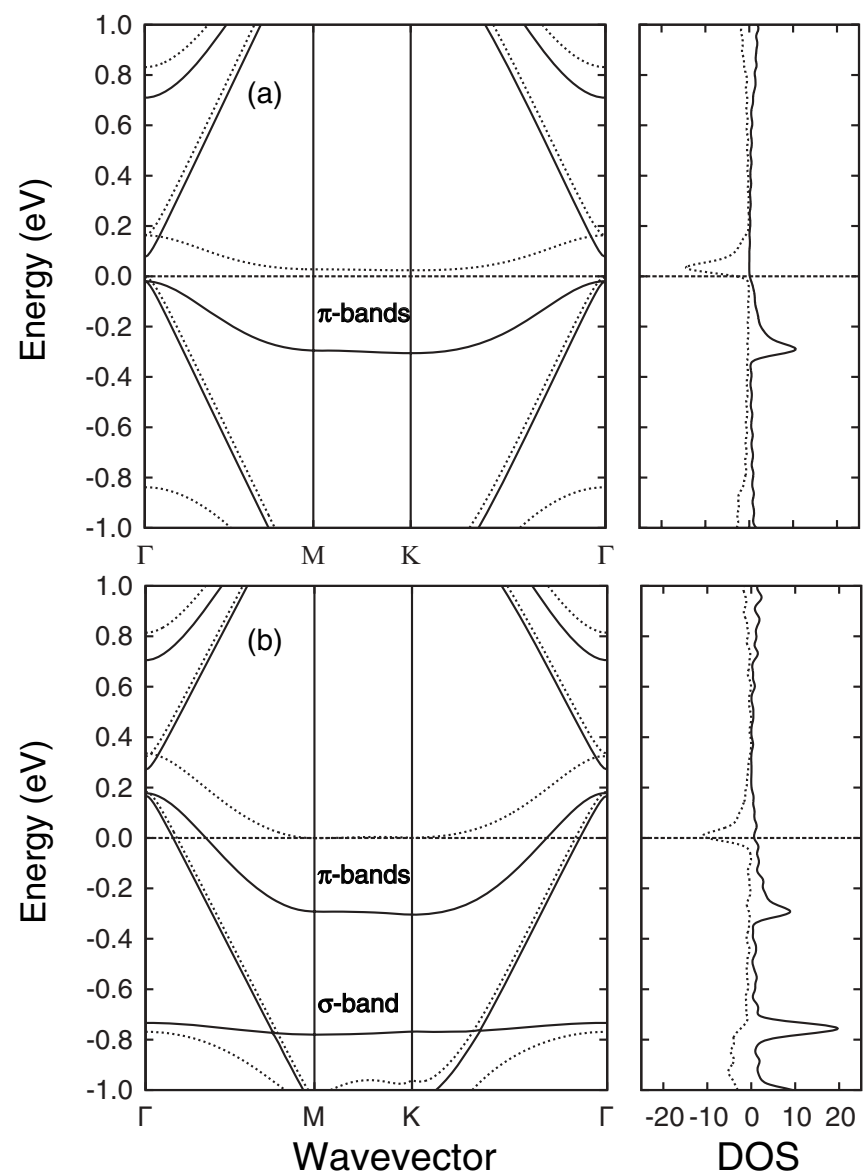

FIG. 2. Calculated spin resolved band structure (left panels) and densities of states (right panels) at a vacancy in a $6 \times 6$ supercell in monolayer graphene. In (a) the three $\sigma$ dangling bonds left by the removal of $\mathrm{C}$ atom are saturated with $\mathrm{H}$ atoms. In (b) a bare vacancy is considered. Full relaxation of the atomic geometry is performed in both cases. Solid and broken lines indicate spin majority and spin minority electronic states, respectively. The zero of energy is at the Fermi level.

These results already show the subtle effect of breaking the bipartite character of the lattice by the relaxation of the atoms around the vacancy which, in turn, induces the breaking of the electron-hole symmetry. In the case of bilayer graphene the situation is expected to be even more subtle since, along with these details, the influence of the bottom layer needs to be taken into consideration. Graphene layers are coupled via van der Waals interactions and, to our knowledge, the existing calculations did not tackle this issue appropriately. Also, as discussed below, the value of the $\pi$ magnetic moment turns out to be quite sensitive to details of the calculation such as size and shape of the supercell, generally decreasing with size. To compound things even further, a recent scanning tunneling spectroscopy (STS) observation of the density of states associated with vacancies created under controlled conditions on the surface layer of graphite has been interpreted as a manifestation of $\pi$ magnetism. ${ }^{25}$ A second reading of the experimental data, however, can also be interpreted otherwise, as the evidence of the absence of magnetism. This has prompted us to question whether vacancy-induced $\pi$ magnetism exists at all. 
Here we present extensive and detailed DFT calculations for vacancies in both monolayer and bilayer graphene, including van der Waals interaction ${ }^{39}$ as implemented in the SIESTA code. ${ }^{40-42}$ Our results indicate that reconstructed vacancies in monolayer and bilayer graphene can host highly localized $\sigma$ magnetic moments of $1 \mu_{B}$ (i.e., one unpaired spin), but that the overall extended $\pi$ magnetism progressively decreases as the size of the supercell increases, extrapolating to zero in the zero-concentration limit. This limit is reached more rapidly when the dangling $\sigma$ bond is saturated with $\mathrm{H}$ (and the $\sigma$ magnetic moment is quenched) and also when the vacancy is created on the bilayer. In the latter no significant differences are appreciated between the two lattice sites in this regard. Calculations for a single vacancy performed with a Green's function methodology ${ }^{43}$ as implemented in the ANT.G code ${ }^{44}$ also yield values of the magnetic moment in the $\pi$ orbitals approaching zero, strengthening our conclusion. On the other hand, as expected, a value of $1 \mu_{B}$ for the $\pi$ magnetic moment is obtained for the unlikely case of a total hydrogenation of the $\sigma$ orbitals which prevents Jahn-Teller reconstruction and recovers Lieb's scenario.

\section{METHODOLOGY}

Most of the calculations reported here have been performed with the SIESTA code which uses a basis of numerical atomic orbitals ${ }^{45}$ and separable ${ }^{46}$ norm conserving pseudopotentials ${ }^{47}$ with partial core corrections. ${ }^{48}$ We have found satisfactory the standard double- $\zeta$ basis with polarization orbitals (DZP) which has been used throughout this work. Also a ghost atom at the vacancy has been included to improve the basis set, although it does not change the DZP results. The convergence of the relevant precision parameters was carefully checked. The real space integration grid had a cutoff of $500 \mathrm{Ryd}$. Of the order of up to $600 k$ points were used in the two-dimensional Brillouin zone sampling using the Monkhorst-Pack $k$-points sampling. Spin resolved calculations are performed in most cases. To accelerate the self-consistency convergence, a polynomial broadening of the energy levels was performed using the method of Methfessel and Paxton, ${ }^{49}$ which is very suitable for systems with a large variation of the density of states in the vicinity of the Fermi level as is the case in our system (see below). Broadening like Fermi-Dirac can be inappropriate and give wrong results. It is worth mentioning that the energy differences between nonmagnetic and magnetic solutions are, in general, small, what requires a very high convergence in all precision parameters and tolerances. To obtain the equilibrium geometry we relaxed all the atoms until the forces acting on them were smaller than $0.01 \mathrm{eV} / \AA$. We obtain for the defect free graphene layer a nearest-neighbor distance of $1.435 \AA$ as compared with the experimental value of $1.42 \AA$. In the bilayer calculation including van der Waals forces the distance between planes is $3.42 \AA$ whereas the experimental one for graphite is $3.35 \AA$. To calculate the geometrical and electronic structure of defects, we use the supercell calculation method with $n \times m$ cells containing the defect for $n$ and $m$ integers and standard unit cell vectors. As a general comment concerning the geometry of the vacancy, we obtain results similar to those reported in the literature; the structure remains planar, two dangling $\sigma$ orbitals rebond in a new weak bond of whose length depends on the supercell size, ranging from $2.05 \AA$ in the $6 \times 6$ case to $1.93 \AA$ in the $15 \times 15$ one. The third $\sigma$ orbital remains nonbonded. In Fig. 1 we show a reconstructed vacancy in the middle of a $5 \times 5$ supercell which, in the calculations, is periodically repeated in two dimensions.

\section{MONOLAYER GRAPHENE}

Although we are interested in the case of an isolated vacancy, we are forced to consider a finite concentration of vacancies on the same sublattice; this is what one actually does in supercell calculations when using electronic structure codes such as SIESTA. As already briefly discussed in the introduction, we have first carried out a $6 \times 6$ supercell calculation in two cases: (i) with $\mathrm{H}$ atoms saturating the $\sigma$ dangling bonds [Fig. 2(a)] and (ii) with no extra $\mathrm{H}$ atoms [Fig. 2(b)]. In the former case, which is not so relevant from an experimental point of view, the full passivation of the three dangling bonds almost completely prevents the reconstruction of the lattice, while in the latter a strong Jahn-Teller distortion takes place (see Fig. 1). Left panels show the band structure and right panels the total density of states (DOS). Only $\pi$ bands are visible in Fig. 2(a) whereas the unsaturated $\sigma$ dangling bond forms a band at $\approx-0.75 \mathrm{eV}$ in Fig. 2(b). This band presents a large spin splitting and is almost flat as corresponds to a highly localized state. On the other hand the splitting of the $\pi$ band is much smaller in both cases and presents a visible dispersion which is due to the always present interaction between vacancies due to the semi-localized character of the $\pi$ state created by the vacancy. ${ }^{50}$ In the DOS the majority spin $\sigma$ peak and the two spin-resolved peaks coming from the $\pi$ state are visible.

For the fully saturated vacancy neither the unoccupied band nor the occupied one crosses the Fermi level. The magnetic moment is thus always quantized to $1 \mu_{B}$ (we have checked that this is the case for any concentration of vacancies), as predicted by Lieb's theorem. Note that the $\sigma$ bonds are saturated and do not host any magnetism here. Note also that the bipartite nature of the lattice is preserved, essentially restoring the electron-hole symmetry. The low-energy physics resulting from these types of vacancies is completely equivalent to the physics of hydrogenated graphene ${ }^{51}$ and has been discussed at length in Ref. 15. For the unsaturated vacancy the band structure presents subtle differences. Both spin-split $\pi$ bands cross the Fermi level, the upper one actually staying pinned to it. This prevents the magnetic moment from reaching the saturation value of $2 \mu_{B}\left(1 \mu_{B}\right.$ from the $\sigma$ bond plus $1 \mu_{B}$ from the vacancy-induced $\pi$ state), yielding a total value of around $1.71 \mu_{B}$ for this particular calculation. This is linked to the remarkable fact that the Fermi level lies below the Dirac point, which is equivalent to saying that the vacancy acts as an acceptor impurity.

We should note at this point that the value of the magnetic moment for the reconstructed vacancy, which is the relevant case from the experimental point of view, changes with the size of the supercell, so we set out now to do a systematic study. Figure 3 shows the band structure for an increasing supercell size sequence $3 n \times 3 n$ up to $15 \times 15$. While the $\sigma$ band becomes quickly completely flat at around $-0.8 \mathrm{eV}$, the 

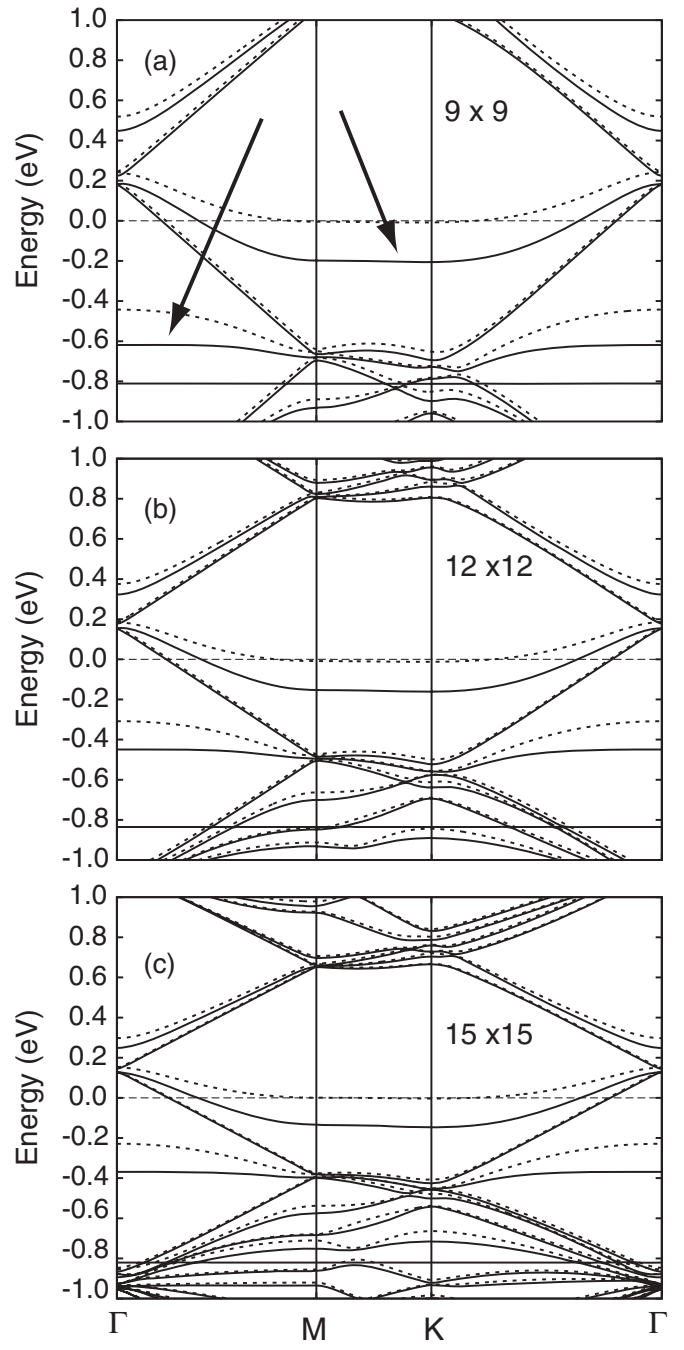

FIG. 3. Calculated spin resolved band structure at a vacancy in increasingly $n \times n$ supercells in monolayer graphene. Panels (a)-(c) stand for $9 \times 9,12 \times 12$, and $15 \times 15$ supercells respectively. Solid and broken lines indicate spin majority and spin minority electronic states. The zero of energy is at the Fermi level. The arrows in panel (a) indicate states induced by the defect [similar bands can be identified in panels (b) and (c)].

$\pi$ band retains the dispersion and the spin splitting although these become flatter and smaller, respectively, as the supercell size increases. This reflects the increasing distance between vacancies and the concomitant increasing extension of the $\pi$ state induced by the vacancy at the Dirac point. (The lattice reconstruction does not allow us to establish a perfect analogy with the Dirac state in the standard tight-binding model which decays as $1 / r$, but we have no reason to expect otherwise). Interestingly, the partially occupied upper spin-split $\pi$ band stays pinned at the Fermi level on a part of the Brillouin zone for all supercell sizes. Also the difference between the Dirac point and the Fermi level decreases, which can be easily understood since the concentration of vacancies (or acceptor impurities) decreases. It is important at this point to notice that the vacancy does not only induce one $\pi$ band around the Fermi

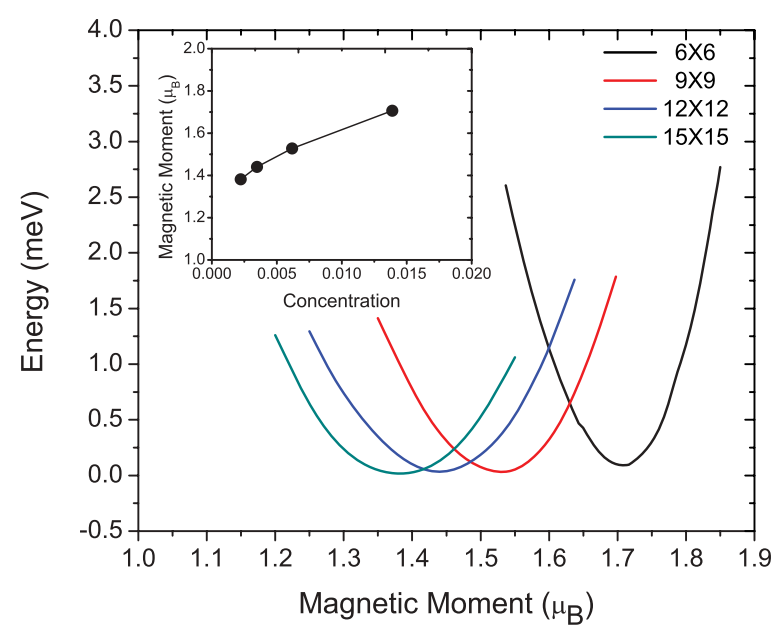

FIG. 4. (Color online) Total energy versus magnetic moment for different supercell sizes for a monovacancy on a graphene monolayer. The inset indicates the magnetic moment at the total energy minimum as a function of the vacancy concentration (inverse of the supercell size).

energy. As indicated by arrows in Fig. 3(a), a new set of $\pi$ bands emerge which carry spectral weight of the resonance mostly in the occupied part of the spectrum. In the zero-concentration limit, this set of bands should merge into a continuum and give a finite width to the resonance in the energy sector of occupied states (see below).

In Fig. 4 we show total energy calculations as a function of fixed magnetic moment $\mu$ for various supercell sizes. One can easily appreciate how, as the supercell size increases, the minimum energy value of $\mu, \mu_{0}$, moves towards $1 \mu_{B}$, which is the lower limit imposed by the unpaired electron of the $\sigma$ dangling bond. At the same time, $d^{2} E(\mu) /\left.d \mu^{2}\right|_{\mu_{0}} \rightarrow 0$, which amounts to a very large susceptibility per vacancy. This shallow variation of the energy with the magnetic moment is a remarkable fact; it should be noticed that, for instance, in the $15 \times 15$ case, the magnetic moment in the $\pi$ states can vary around $0.4 \mu_{B}$ within $1 \mathrm{meV}$. This indicates that, even at low temperatures, the magnetic moment is ill defined. This is even more pronounced in the bilayer case (see below). The dependence of $\mu_{0}$ on the inverse of the supercell size (i.e., the concentration of impurities) is plotted in the inset of Fig. 4 (see below for further analysis).

We finally examine the possibility of having the dangling $\sigma$ bond saturated with atomic $\mathrm{H}$. The calculations are performed allowing relaxations of all the atoms as indicated above. Now the $\sigma$ band disappears from the energy window of interest (see Fig. 5) along with the associated magnetic moment. While for small supercells (or high concentrations of vacancies) the spin splitting of the $\pi$ band is still visible, it already completely vanishes for supercell sizes as those considered in the previous case. This result reflects the importance of considering the mutual influence between the $\sigma$ and $\pi$ electrons, at least as far as magnetic properties is concerned, when the former are not part of a bond to other species such as, e.g., H. This effect cannot be captured by the Hubbard model where the saturation of the sigma bonds is always implied even if the hopping terms are adapted to the atomic reconstruction. ${ }^{52}$ 

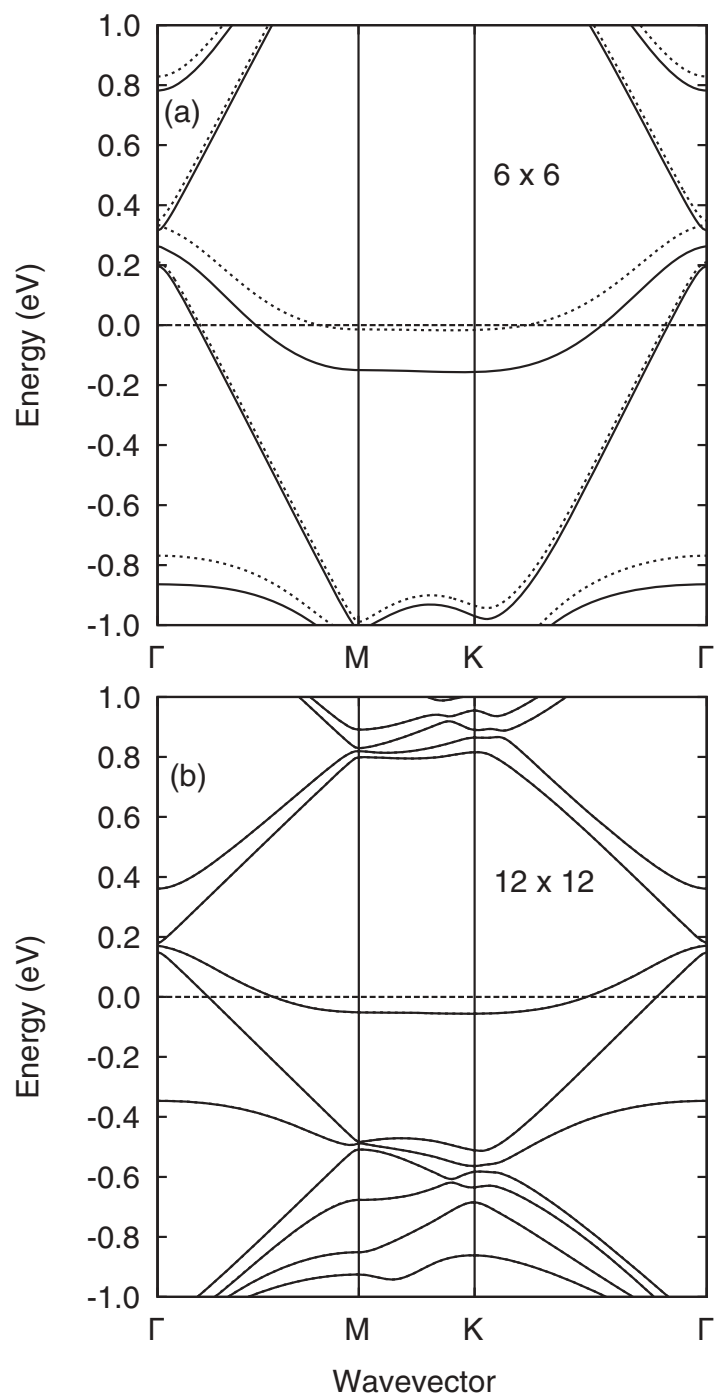

FIG. 5. Band structure when the $\sigma$ dangling orbital of the vacancy is saturated with a $\mathrm{H}$ atom for a (a) $6 \times 6$ and a (b) $12 \times 12$ supercell. Solid and broken lines indicate spin majority and spin minority electronic states. The zero of energy is at the Fermi level.

\section{BILAYER GRAPHENE}

We now consider vacancies on bilayer graphene with Bernal stacking. In this situation, removing a $\mathrm{C}$ atom from one sublattice or the other is different due to the underlying graphene layer, resulting in two types of vacancies, $\alpha$ and $\beta$, depending on whether or not the vacancy is created on top or in a hollow position. ${ }^{25}$ The band structure of a vacancy in a $9 \times 9$ supercell in both cases is shown in Fig. 6. As in the previous case, we allow for full relaxation of the atomic coordinates on the layer containing the vacancy. Figure 6(c) shows the bands for a bilayer without vacancies. The mass acquired by the Dirac electrons (the parabolic dispersion at the Fermi energy) as a result of the interlayer interactions is evident in the plot. The $\pi$ bands associated with the vacancy, regardless of the sublattice creation site, are spin-split for small cells. Contrary to the monolayer case, the minority spin band crosses the Fermi level, already indicating a stronger tendency towards the quenching of $\pi$ magnetism than in the monolayer. Therefore, it

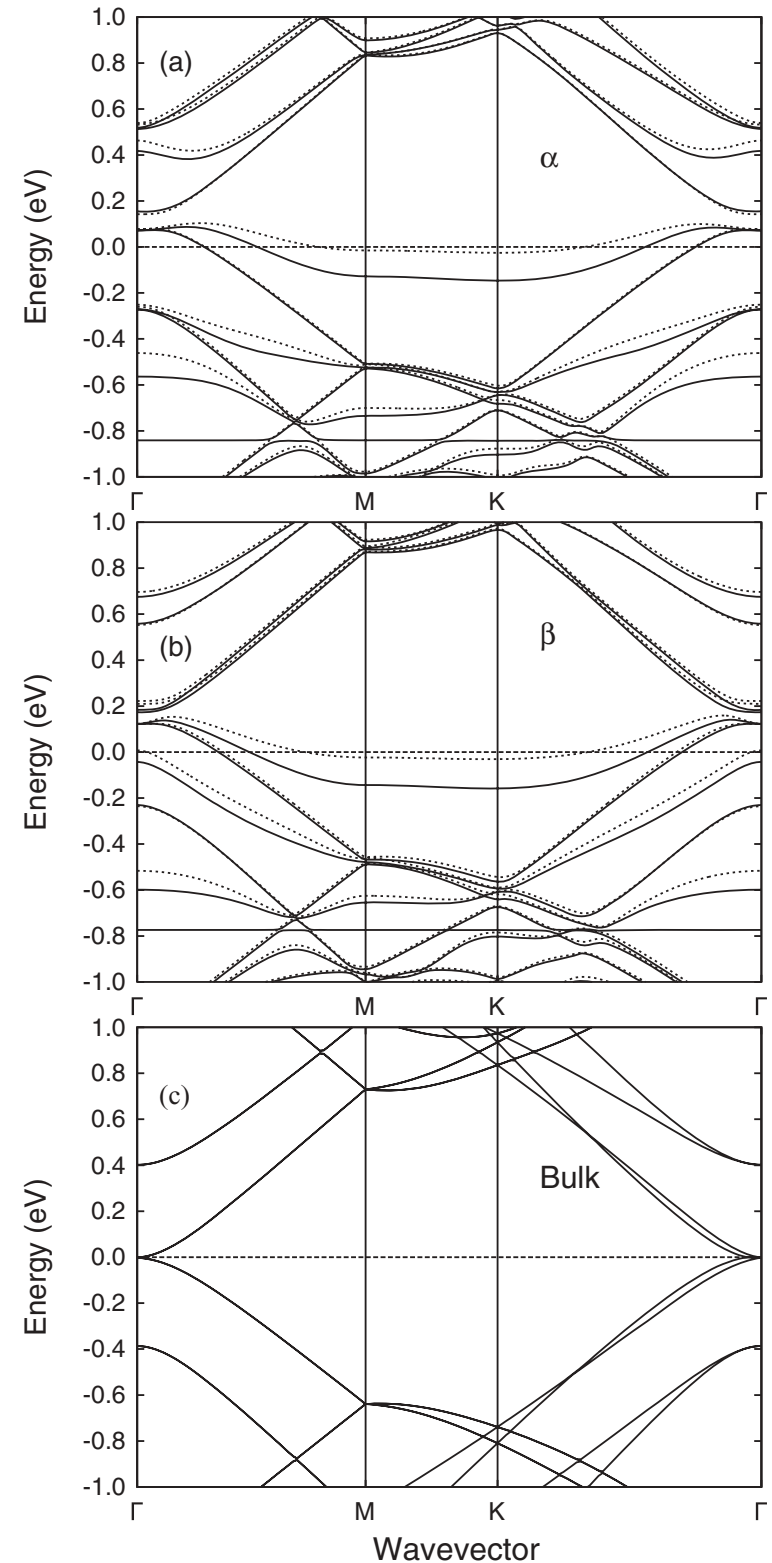

FIG. 6. Band structure for vacancies on a bilayer for a $9 \times 9$ supercell. Solid and broken lines indicate spin majority and spin minority electronic states. The zero of energy is at the Fermi level. Panels (a) and (b) refer to a vacancy created on top $(\alpha)$ or hollow $(\beta)$ positions, respectively. Panel (c) represents the defect free graphene bilayer.

should not come as a surprise that, as in the monolayer case, the spin splitting goes to zero as the distance between vacancies increases, remaining only the magnetic moment associated with the $\sigma$ bond. The difference between the $\alpha$ and $\beta$ cases is minor. The bands in the $\alpha$ case are narrower than those of the $\beta$ case as expected. ${ }^{25}$ The inset in Fig. 7 shows $\mu_{0}$ as a function of the inverse of the supercell size for $\alpha$ vacancies. Compared to the monolayer result in Fig. 4, one can safely extrapolate $\mu_{0} \rightarrow 1 \mu_{B}$ in the zero-concentration limit. Note that the somewhat erratic behavior of $\mu_{0}$ as a function of the inverse supercell size can be attributed to considering all consecutive sizes, while in the monolayer case we are only plotting results for $3 n \times 3 n$ supercells. In light of the results, 


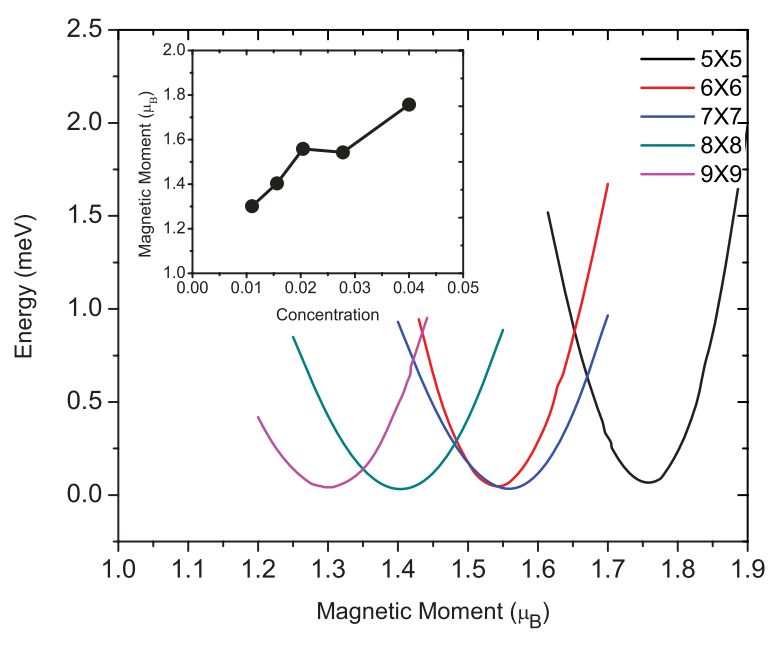

FIG. 7. (Color online) Total energy versus magnetic moment for different supercell sizes for an $\alpha$ vacancy in a graphene bilayer. The inset shows the magnetic moment at the total energy minimum as a function of the vacancy concentration (inverse of the supercell size).

as for the monolayer, we also expect $\mu_{0} \rightarrow 0 \mu_{B}$ if the $\sigma$ dangling bond is saturated. Also, as in the monolayer case, we obtain shallow energy curves versus magnetic moment, indicating an even higher susceptibility (see Fig. 7).

\section{CRITICAL ANALYSIS OF RESULTS AND FINAL CONSIDERATIONS}

While the results for the bilayer vacancy and the monolayer vacancy with $\mathrm{H}$ seem conclusive regarding the vanishing value of the $\pi$ magnetism in the low concentration limit, the ones for the $\mathrm{H}$ free monolayer vacancy remain less clear. We would like to discard any possible influence on the results of the specific sequence of supercells considered in our calculations, so we have also performed additional calculations with supercells out of the main sequence $3 n \times 3 n$. We now plot in Fig. 8 all the results for the total magnetic moment, including values obtained with all types of supercells. In light of this plot we can

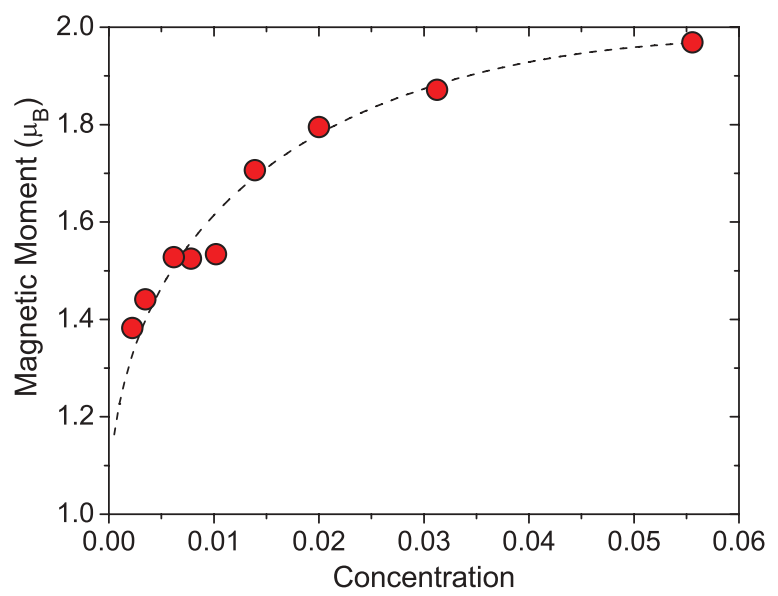

FIG. 8. (Color online) Calculated magnetic moment induced by a vacancy in a graphene monolayer for various concentrations (inverse of the supercell size). The red circles are the calculated values, and the broken line is a fit to $1+a n+b \sqrt{n}$. safely conclude that $\mu_{0}$ goes to $1 \mu_{B}$ in the zero-concentration limit $n \rightarrow 0$, possibly as $\propto n^{\delta}$ where $\delta<1$. In fact, despite that the behavior of $\mu_{0}$ is not monotonic, a good fit to $\mu_{0}(n)=$ $1+a n+b \sqrt{n}$ can be done $(a=-15.11, b=7.64)$.

We would like to address now the influence of the periodicity on the results. To this aim, we have also performed calculations for truly isolated vacancies with the help of the ALACANT package, ${ }^{44}$ in particular, we have employed our code ANT.G which interfaces with GAUSSIAN09. ${ }^{53}$ In this case the supercell is surrounded by an effective medium defined by a two-dimensional Bethe lattice ${ }^{54}$ of coordination three and Slater-Koster parameters for the C $s p$ orbitals. Here the Green's function of the supercell is self-consistently computed subject to a fixed self energy representing the Bethe lattice. In contrast to the calculations with SIESTA, the vacancy is here truly isolated, but the electronic structure outside the cell remains fixed and unmagnetized. Unlike bulk graphene, the Bethe lattice model presents a finite density of states at the Fermi energy, which gives the quasilocalized $\pi$ state of the vacancy a finite lifetime for any cell size even at zero energy. We have also used here the generalized gradient approximation through the BPBE functional as implemented in GAUSSIAN $09^{53}$ and a basis set equivalent to that in the SIESTA calculations. The atomic structure has also been optimized, obtaining essentially the same geometry. The values of the magnetic moments so obtained are all in the range $\approx 1.1-1.3 \mu_{B}$, with a clear trend towards $1.0 \mu_{B}$ as the system size increases. One may conclude that the periodicity, if anything, enhances the values of the $\pi$ magnetic moments induced by the vacancy.

To make connection with available experimental information ${ }^{25}$ we have plotted in Fig. 9 the DOS projected on the $\pi$ orbital of the vacancy atom with the dangling bond (for the other two vacancy atoms the results are similar). Since we have shown that in the limit of an isolated vacancy the $\pi$ magnetism vanishes, the calculation is here a non spin resolved one for an $18 \times 18$ supercell. We obtain an asymmetric and almost fully occupied sharp-peaked resonance at the Fermi level, its spectral shape strongly deviating from a symmetric Breit-Wigner or $1 /|E|$ resonance. ${ }^{50}$ Most of its weight is in the valence band with no extra structure in the conduction band and a small gap right above the main peak. This anomalous form of the line shape is a dramatic consequence of the electron-hole symmetry breaking (see qualitatively similar results in a model calculation by Pereira et al..$^{50}$ ). The asymmetry and the presence of the small gap right above the sharp peak would prevent, in the isolated vacancy limit, the Stoner instability and the formation of an extended magnetic moment.

From these results several conclusions can be extracted regarding various experimental observations:

(i) Our results indicate that only a high concentration of ordered vacancies on the same sublattice can sustain finite values of the $\pi$ magnetic moments and lead to a ferromagnetically ordered state. The concentration below which these magnetic moments disappear depends on whether or not the $\sigma$ dangling bond is passivated, being much higher for the passivated case. In addition, one should not forget that, on average, the same number of vacancies are expected on both sublattices. In this case the $\pi$ magnetic moments will be coupled antiferromagnetically or even quenched when vacancies are in proximity, ${ }^{15}$ further disfavoring the observation of 


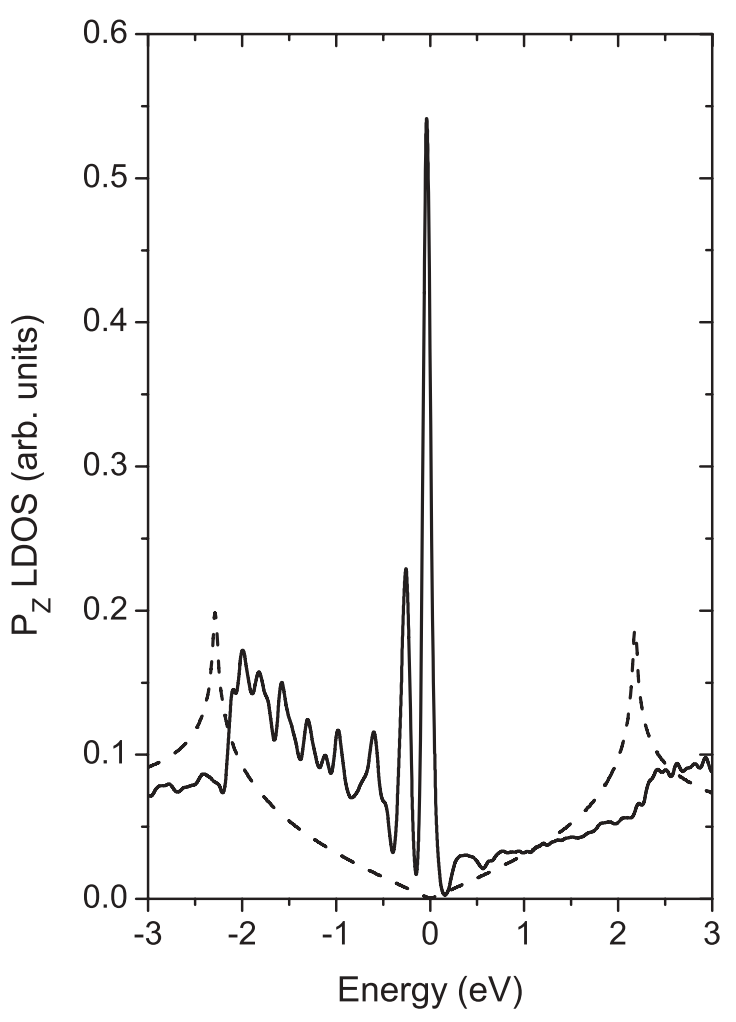

FIG. 9. Density of states projected on the $\pi$ orbital at an atom in the vacancy (solid line) in the nonmagnetic solution. A small $(0.05 \mathrm{eV})$ Gaussian broadening has been included for presentation purposes. The corresponding density of states in a defect free graphene is represented by the broken line.

ferromagnetism. On top of that, an excessive concentration of vacancies will likely render graphene unstable.

(ii) Although one should keep in mind that the STS results by Brihuega et al..$^{25}$ refer to surface graphite, our results are compatible with their observations without invoking the existence of $\pi$ magnetism. In their experiment no trace of two spin-split peaks near the Fermi energy can be seen. Furthermore, although the DOS in Fig. 9 corresponds to a graphene monolayer, the asymmetry or shoulder that appears in the experimental $d I / d V$ peak at negative bias nicely compares with our result. We should note, nevertheless, that we obtain a large magnetic susceptibility mainly associated to the soft position of the spin-majority peak in the DOS. The possibility for thermal fluctuations to wash out this peak from the DOS, masking the spin-split structure, cannot be entirely ruled out.

(iii) In addition to the disappearance of the $\pi$ magnetic moments and as shown in Fig. 3, the $\sigma$ band becomes rapidly flat as the concentration of vacancies decreases. This indicates that the localized $\sigma$ magnetic moments do not interact for any reasonable concentration and, therefore, unsaturated vacancies should behave as paramagnetic centers. Upon completion of this work an experiment by Nair et al..$^{55}$ has unambiguously shown paramagnetic behavior of irradiated graphene, indicating that the vacancies so created present magnetic moments but no magnetic order. This experiment is consistent with our results for unsaturated vacancies. Nevertheless, one should not forget that the passivation of the sigma bond will strongly depend on the experimental conditions under which the vacancies are created.

(iv) To conclude, one should keep in mind that $\pi$ magnetism and magnetic order can still emerge through atomic $\mathrm{H}$ adsorption or through any other adsorbate capable of similar covalent bonding to $p_{z}$ orbitals. This magnetism should be amenable to experimental verification, for instance in magnetotransport measurements, as recently proposed..$^{56,57}$

\section{ACKNOWLEDGMENTS}

We are indebted to I. Brihuega, J. M. Gómez-Rodríguez, and M. M. Ugeda for discussions and helpful comments concerning their experimental results. We also appreciate discussions with G. Gómez-Santos and J. M. Soler. This work has been financially supported by MICINN of Spain under Grants No. FIS2009-12712, No. FIS2010-21883, No. MAT07-67845, No. CONSOLIDER CSD2007-00010, and No. CONSOLIDER CSD2007-00050.
${ }^{1}$ M. Fujita, K. Wakabayashi, K. Nakada, and K. Kusakabe, J. Phys. Soc. Jpn. 65, 1920 (1996).

${ }^{2}$ K. Nakada, M. Fujita, G. Dresselhaus, and M. S. Dresselhaus, Phys. Rev. B 54, 17954 (1996).

${ }^{3}$ K. Wakabayashi, M. Fujita, H. Ajiki, and M. Sigrist, Phys. Rev. B 59, 8271 (1999).

${ }^{4}$ T. Hikihara, X. Hu, H.-H. Lin, and C.-Y. Mou, Phys. Rev. B 68, 035432 (2003).

${ }^{5}$ K. Kusakabe and M. Maruyama, Phys. Rev. B 67, 092406 (2003).

${ }^{6}$ Y.-W. Son, M. L. Cohen, and S. G. Louie, Nature (London) 444, 347 (2006).

${ }^{7}$ J. Fernández-Rossier and J. J. Palacios, Phys. Rev. Lett. 99, 177204 (2007).

${ }^{8}$ D.-e. Jiang, B. G. Sumpter, and S. Dai, J. Chem. Phys. 127, 124703 (2007).
${ }^{9}$ O. Hod, V. Barone, and G. E. Scuseria, Phys. Rev. B 77, 035411 (2008).

${ }^{10}$ M. Ezawa, Phys. Rev. B 76, 245415 (2007).

${ }^{11}$ A. A. El-Barbary, R. H. Telling, C. P. Ewels, M. I. Heggie, and P. R. Briddon, Phys. Rev. B 68, 144107 (2003).

${ }^{12}$ P. O. Lehtinen, A. S. Foster, Y. Ma, A. V. Krasheninnikov, and R. M. Nieminen, Phys. Rev. Lett. 93, 187202 (2004).

${ }^{13}$ M. A. H. Vozmediano, M. P. López-Sancho, T. Stauber, and F. Guinea, Phys. Rev. B 72, 155121 (2005).

${ }^{14}$ O. V. Yazyev and L. Helm, Phys. Rev. B 75, 125408 (2007).

${ }^{15}$ J. J. Palacios, J. Fernández-Rossier, and L. Brey, Phys. Rev. B 77, 195428 (2008).

${ }^{16}$ R. Singh and P. Kroll, J. Phys.: Condens. Matter 21, 196002 (2009).

${ }^{17}$ W. Li, M. Zhao, X. Zhao, Y. Xia, and Y. Mu, PhysChemChemPhys 12, 13699 (2010). 
${ }^{18}$ L. Pisani, B. Montanari, and N. M. Harrison, New J. Phys. 10, 033002 (2008).

${ }^{19}$ M. W. C. Dharma-wardana and M. Z. Zgierski, Phys. E (Amsterdam, Neth.) 41, 80 (2008).

${ }^{20}$ J. Červenka, M. I. Katsnelson, and C. F. J. Flipse, Nat. Phys. 5, 840 (2009).

${ }^{21}$ P. Esquinazi, A. Setzer, R. Höhne, C. Semmelhack, Y. Kopelevich, D. Spemann, T. Butz, B. Kohlstrunk, and M. Lösche, Phys. Rev. B 66, 024429 (2002).

${ }^{22}$ J. Barzola-Quiquia, P. Esquinazi, M. Rothermel, D. Spemann, T. Butz, and N. García, Phys. Rev. B 76, 161403 (2007).

${ }^{23}$ Y. Wang, Y. Huang, Y. Song, X. Zhang, Y. Ma, J. Liang, and Y. Chen, Nano Lett. 9, 220 (2009).

${ }^{24}$ A. Candini, C. Alvino, W. Wernsdorfer, and M. Affronte, Phys. Rev. B 83, 121401 (2011).

${ }^{25}$ M. M. Ugeda, I. Brihuega, F. Guinea, and J. M. Gómez-Rodríguez, Phys. Rev. Lett. 104, 096804 (2010).

${ }^{26}$ C. Tao, L. Jiao, O. V. Yazyev, Y.-C. Chen, J. Feng, X. Zhang, R. B. Capaz, J. M. Tour, A. Zettl, S. G. Louie, H. Dai, and M. F. Crommie, Nat. Phys. 7, 616 (2011).

${ }^{27}$ D. Martínez-Martín, M. Jaafar, R. Pérez, J. Gómez-Herrero, and A. Asenjo, Phys. Rev. Lett. 105, 257203 (2010).

${ }^{28}$ E. H. Lieb, Phys. Rev. Lett. 62, 1201 (1989).

${ }^{29}$ M. M. Ugeda, I. Brihuega, F. Hiebel, P. Mallet, J.-Y. Veuillen, J. M. Gomez-Rodriguez, and F. Yndurain, Phys. Rev. B 85, 121402(R) (2012).

${ }^{30}$ T. Wassmann, A. P. Seitsonen, A. M. Saitta, M. Lazzeri, and F. Mauri, Phys. Rev. Lett. 101, 096402 (2008).

${ }^{31}$ P. Koskinen, S. Malola, and H. Häkkinen, Phys. Rev. Lett. 101, 115502 (2008).

${ }^{32}$ J. Kunstmann, C. Özdogan, A. Quandt, and H. Fehske, Phys. Rev. B 83, 045414 (2011).

${ }^{33}$ J. Tian, H. Cao, W. Wu, Q. Yu, and Y. P. Chen, Nano Lett. 11, 3663 (2011).

${ }^{34}$ H. Kumazaki and D. S. Hirashima, J. Phys. Soc. Jpn. 76, 034707 (2007).

${ }^{35}$ S. Choi, B. W. Jeong, S. Kim, and G. Kim, J. Phys.: Condens. Matter 20, 235220 (2008).

${ }^{36}$ E. V. Castro, M. P. López-Sancho, and M. A. H. Vozmediano, Phys. Rev. Lett. 104, 036802 (2010).

${ }^{37}$ R. Faccio, H. Pardo, P. A. Denis, R. Y. Oeiras, F. M. Araújo-Moreira, M. Veríssimo-Alves, and A. W. Mombrú, Phys. Rev. B 77, 035416 (2008).

${ }^{38}$ B. R. K. Nanda, M. Sherafati, Z. Popović, and S. Satpathy, arXiv:1105.1129.

${ }^{39}$ M. Dion, H. Rydberg, E. Schroder, D. C. Langreth, and B. I. Lundqvist, Phys. Rev. Lett. 92, 246401 (2004).

${ }^{40}$ J. M. Soler, E. Artacho, J. D. Gale, A. Garcia, J. Junquera, P. Ordejon, and D. Sanchez-Portal, J. Phys.: Condens. Matter 14, 2745 (2002).
${ }^{41}$ P. Ordejón, E. Artacho, and J. M. Soler, Phys. Rev. B 53, R10441 (1996).

${ }^{42}$ G. Román-Pérez and J. M. Soler, Phys. Rev. Lett. 103, 096102 (2009).

${ }^{43}$ Bor-Luen Huang, Ming-Che Chang, and Chung-Yu Mou, Phys. Rev. B 82, 155462 (2010); Bor-Luen Huang and Chung-Yu Mou, Europhys. Lett. 88, 68005 (2009).

${ }^{44}$ J. J. Palacios, D. Jacob, A. J. Pérez-Jiménez, E. S. Fabián, E. Louis, and J. A. Vergés, ALACANT package (http://alacant.dfa.ua.es).

${ }^{45}$ O. F. Sankey and D. J. Niklewski, Phys. Rev. B 40, 3979 (1989).

${ }^{46}$ L. Kleinman and D. M. Bylander, Phys. Rev. Lett. 48, 1425 (1982).

${ }^{47}$ N. Troullier and J. L. Martins, Phys. Rev. B 43, 1993 (1991).

${ }^{48}$ S. G. Louie, S. Froyen, and M. L. Cohen, Phys. Rev. B 26, 1738 (1982).

${ }^{49}$ M. Methfessel and A. T. Paxton, Phys. Rev. B 40, 3616 (1989).

${ }^{50}$ V. M. Pereira, F. Guinea, J. M. B. LopesdosSantos, N. M. R. Peres, and A. H. CastroNeto, Phys. Rev. Lett. 96, 036801 (2006).

${ }^{51}$ D. Soriano, F. Muñoz-Rojas, J. Fernández-Rossier, and J. J. Palacios, Phys. Rev. B 81, 165409 (2010).

${ }^{52}$ M. P. López-Sancho, F. de Juan, and M. A. H. Vozmediano, Phys. Rev. B 79, 075413 (2009).

${ }^{53}$ M. J. Frisch, G. W. Trucks, H. B. Schlegel, G. E. Scuseria, M. A. Robb, J. R. Cheeseman, G. Scalmani, V. Barone, B. Mennucci, G. A. Petersson, H. Nakatsuji, M. Caricato, X. Li, H. P. Hratchian, A. F. Izmaylov, J. Bloino, G. Zheng, J. L. Sonnenberg, M. Hada, M. Ehara, K. Toyota, R. Fukuda, J. Hasegawa, M. Ishida, T. Nakajima, Y. Honda, O. Kitao, H. Nakai, T. Vreven, J. A. Montgomery Jr., J. E. Peralta, F. Ogliaro, M. Bearpark, J. J. Heyd, E. Brothers, K. N. Kudin, V. N. Staroverov, R. Kobayashi, J. Normand, K. Raghavachari, A. Rendell, J. C. Burant, S. S. Iyengar, J. Tomasi, M. Cossi, N. Rega, J. M. Millam, M. Klene, J. E. Knox, J. B. Cross, V. Bakken, C. Adamo, J. Jaramillo, R. Gomperts, R. E. Stratmann, O. Yazyev, A. J. Austin, R. Cammi, C. Pomelli, J. W. Ochterski, R. L. Martin, K. Morokuma, V. G. Zakrzewski, G. A. Voth, P. Salvador, J. J. Dannenberg, S. Dapprich, A. D. Daniels, Ö. Farkas, J. B. Foresman, J. V. Ortiz, J. Cioslowski, and D. J. Fox, Gaussian 09 revision a.1 (Gaussian Inc., Wallingford, CT, 2009).

${ }^{54}$ J. D. Joannopoulos and F. Yndurain, Phys. Rev. B 10, 5164 (1974).

${ }^{55}$ R. R. Nair, M. Sepioni, I. Tsai, O. Lehtinen, J. Keinonen, A. V. Krasheninnikov, T. Thomson, A. K. Geim, and I. V. Grigorieva, Nat. Phys. 8, 199 (2012).

${ }^{56}$ D. Soriano, N. Leconte, P. Ordejón, J.-C. Charlier, J.-J. Palacios, and S. Roche, Phys. Rev. Lett. 107, 016602 (2011).

${ }^{57}$ N. Leconte, D. Soriano, S. Roche, P. Ordejon, J.-C. Charlier, and J. J. Palacios, ACS Nano 5, 3987 (2011). 